\title{
A BIPHASIC ANAPHYLACTIC REACTION DURING PLASMAPHERESIS IN A NON-ATOPIC PATIENT WITH GUILLAIN-BARRÉ SYNDROME
}

\author{
Sule Akin Enes, Anis Aribogan, Asli Karsli \\ Department of Anesthesiology and Reanimation, Ankara, Turkey
}

Purpose of the study:

Plasmapheresis is a preferable therapeutic method in Guillain-Barré syndrome (GBS).In atopic patients, there is a risk of anaphylactic shock in plasmapheresis

In this case report, a biphasic anaphylactic reaction is presented in a patient with no allergy history who underwent plasmapheresis with diagnosis of GBS.

\section{Materyal and Methods:}

Plasmapheresis was planned in a 50-year-old male patient with GBS.

During the plasmapheresis no complication was seen except a treatable hypotension and itching in the final session.

Five hours after the final session, he admitted to ICU with a severe dyspnea.

He has no history of allergy or asthma but bronchoconstriction was typical at the physical examination.

At that moment, severe hypotension, loss of consciousness and skin rashes developed in the dyspneic patient.

Clinic progression was considered as biphasic anaphylactic reaction and $0.5 \mathrm{mg}$ of epinephrine was administered intramuscularly. Fluid therapy and high flow oxygen with mask were added.

Epinephrine was reapplied in five minutes due to ineffective hemodynamic response.

Consciousness and normal vital signs were achieved without necessity of cardiopulmonary resuscitation after this dose and complete recovery was maintained in 24 hours.

\section{Results:}

Biphasic anaphylactic reactions may develope in 2-72 hours after the primary anaphylactoid reaction.

Our patient had no history of allergy but late-onset lifethreatening vital signs suggested severe biphasic anaphylactic reaction following the hypotension attack during plasmapheresis.

Epinephrine administrations were indicated according to the recommendations of the ERC guidelines for preventing a sudden cardiac arrest and improving vital findings

\section{Result:}

Biphasic anaphylactic reactions may develope in 2-72 hours after the primary anaphylactoid reaction.

Our patient had no history of allergy but late-onset lifethreatening vital signs suggested severe biphasic anaphylactic reaction following the hypotension attack during plasmapheresis.

Epinephrine administrations were indicated according to the recommendations of the ERC guidelines for preventing a sudden cardiac arrest and improving vital findings

status.

We considered that all the anesthesiologist in our department had taken ERC courses which was very important in successful resuscitation of the patient.

\section{Conclusion:}

We emphasize that even non-atopic patients can have a risk of biphasic anaphylactic reaction as a life-threatening situation during plasmapheresis Using ERC guidelines for treatment of these special cardiopulmonary resuscitation circumstances should be a life saving approach.

\section{References:}

Anaesthesiol. Intensive Ther 2013,45:7-13

Immunol Allergy Clin North Am: 2007,27: 309-326 